\title{
Diagnosing Pulmonary Embolism in Pregnancy: Are Biomarkers and Clinical Predictive Models Useful?
}

\author{
Barbara V. Parilla, MD ${ }^{1}$ Rachel Fournogerakis, $\mathrm{DO}^{1}$ Amy Archer, $\mathrm{MD}^{2}$ Suela Sulo, $\mathrm{PhD}^{3}$ \\ Lisa Laurent, $\mathrm{MD}^{4}$ Patricia Lee, $\mathrm{MD}^{5}$ Benazir Chhotani, MD ${ }^{5}$ Kathleen Hesse, RN ${ }^{6}$ Erik Kulstad, MD \\ ${ }^{1}$ Department of Obstetrics and Gynecology, Advocate Lutheran \\ General Hospital, Park Ridge, Illinois \\ 2 Department of Emergency Medicine, Advocate Lutheran General \\ Hospital, Park Ridge, Illinois \\ 3 James R. \& Helen D. Russell Institute for Research \& Innovation, \\ Advocate Lutheran General Hospital, Park Ridge, Illinois \\ ${ }^{4}$ Department of Radiology, Advocate Lutheran General Hospital, \\ Park Ridge, Illinois \\ ${ }^{5}$ Department of Emergency Medicine, Advocate Illinois Masonic \\ Medical Center, Chicago, Illinois \\ ${ }^{6}$ Department of Emergency Medicine, Advocate Christ Medical \\ Center, Oak Lawn, Illinois \\ Address for correspondence Barbara V. Parilla, MD, Department of \\ Obstetrics and Gynecology, Advocate Lutheran General Hospital, \\ 1875 W Dempster St, Suite 325, Park Ridge, IL 60068 \\ (e-mail: Barbara.Parilla@advocatehealth.com).
}

Am J Perinatol Rep 2016;6:e160-e164.

\begin{abstract}
Keywords

- pulmonary embolism

- pregnancy

- modified Wells score

- D-dimer

- brain

- natriuretic protein

Objective The objective of this study was to evaluate whether trimester-specific D-dimer levels or the modified Wells score (MWS) is a useful risk stratification tool to exclude pregnant women at low risk of pulmonary embolism (PE) from diagnostic imaging.

Study Design This is a prospective and retrospective cohort study. Pregnant women who underwent diagnostic imaging for suspected PE were prospectively enrolled. D-dimer serum levels were drawn, and a MWS was assigned. Pregnant women diagnosed with a PE before study launch who underwent diagnostic imaging and had a D-dimer level drawn were also evaluated.

Results In this study, 17 patients were diagnosed with a PE and 42 patients had no PE on diagnostic imaging. Sixteen out of 17 patients with a PE versus 11 out of 42 without PE had an abnormal D-dimer level $(p=0.001)$. Four patients with a PE versus zero without a PE had an abnormal MWS $(p=0.005)$. The combination of a trimester-specific D-dimer level along with the MWS was abnormal in all 17 patients with a documented PE versus $11 / 42(26.2 \%)$ patients without a documented PE $(p=0.001)$.

Conclusion A combination of trimester-specific D-dimer levels along with a MWS can be used in pregnancy to triage women into a low-risk category for PE and thereby avoid radiation exposure in a majority of pregnant patients.
\end{abstract}

Pulmonary embolism (PE) continues to be a major cause of maternal death in the United States. The diagnosis of PE is challenging in pregnancy as "shortness of breath" and "difficulty breathing" are not uncommon complaints due to normal physiologic changes in pregnancy. ${ }^{1}$ In the nonpregnant population, validated clinical predictive models, such as the modified Wells score (MWS), ${ }^{2}$ (- Table 1) stratify "likely PE" from "unlikely PE" to exclude patients from potentially received

February 18, 2016 accepted after revision February 25, 2016
DOI http://dx.doi.org/ 10.1055/s-0036-1582136. ISSN 2157-6998.
Copyright $\odot 2016$ by Thieme Medical Publishers, Inc., 333 Seventh Avenue, New York, NY 10001, USA. Tel: +1(212) 584-4662.
License terms

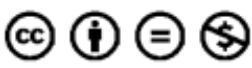


harmful diagnostic imaging. ${ }^{3}$ Combined with a D-dimer $<0.50 \mu \mathrm{g} / \mathrm{mL}$, the negative predictive value of this approach has been found to be $99.5 \%{ }^{4}$ However, D-dimer levels naturally increase in pregnancy, such that the majority of pregnant women have levels above the discriminatory cutoff used to exclude PE outside of pregnancy. ${ }^{5,6}$ Although there are recognized trimester-specific D-dimer levels for pregnancy, ${ }^{7}$ ( - Table 2) they have not yet been assessed as a means to exclude PE in pregnancy. The number of pregnant women who are imaged to exclude PE as a result of the above-mentioned symptoms is high, as clinicians fear fatal misdiagnosis.

In addition to D-dimer, another biomarker, brain natriuretic peptide (BNP) may be useful for risk stratification of PE in pregnancy. BNP is elevated in cardiac damage and failure. Levels double during pregnancy but remain stable irrespective of gestational age (GA). ${ }^{8}$ BNP was found to be useful in the detection of acute PE in postsurgical patients, where D-dimer levels are frequently elevated and therefore not discriminatory. ${ }^{9}$ Finally, increased levels of BNP have been shown to be a marker for right ventricle dysfunction, and higher levels correlate with an increase in morbidity and mortality in acute PE. ${ }^{10}$

The purpose of this study was to determine whether trimester-specific D-dimer and BNP serum levels in combination with the MWS can be used to reliably stratify pregnant patients into a low-risk category. This may decrease the number of computed tomography pulmonary angiography (CTPA) and ventilation perfusion (VQ) scans performed on pregnant women; thus, resulting in a substantial cost savings to the health care system and decreasing radiation exposure to women and their fetuses.

\section{Materials and Methods}

The study was approved by the facility's Institutional Review Board. Forty-five (76.3\%) pregnant women who presented to the Emergency Department or Labor and Delivery unit at the three participating hospitals (three community teaching hospitals that are part of the largest health care system in Illinois) with signs and symptoms suspicious for PE and who underwent diagnostic imaging (CTPA or VQ scan) were prospectively enrolled in the study. The recruitment period

Table 1 Modified Wells score

\begin{tabular}{|l|l|}
\hline Clinical symptoms of DVT & 3 \\
\hline Other diagnosis less likely than PE & 3 \\
\hline Pulse $>100$ & 1.5 \\
\hline Immobilization $\geq 3$ d or surgery in previous 4 wk & 1.5 \\
\hline Previous DVT or PE & 1.5 \\
\hline Hemoptysis & 1 \\
\hline Malignancy & 1 \\
\hline MWS $\leq 4 \rightarrow$ PE unlikely & \\
MWS $>4 \rightarrow$ PE likely & \\
\hline
\end{tabular}

Abbreviations: DVT, deep vein thrombosis; MWS, modified Wells score; $\mathrm{PE}$, pulmonary embolism.
Table 2 Normal D-dimer levels

\begin{tabular}{|l|l|}
\hline Trimester & D-dimer $(\mu \mathrm{g} / \mathrm{mL})$ \\
\hline First (1-13 wk) & $<0.95$ \\
\hline Second $(14-26 \mathrm{wk})$ & $<1.29$ \\
\hline Third $(27-40 \mathrm{wk})$ & $<1.7$ \\
\hline
\end{tabular}

Source: Abbassi-Ghanavati et al (2009). ${ }^{7}$

was February 2014 to August 2015. The decision to proceed with diagnostic imaging was left up to the individual managing physician. Patients undergoing diagnostic imaging, who gave informed consent for study participation, had serum assays for D-dimer and BNP drawn. Two of the 45 consented patients did not have D-dimer levels drawn, and 1 patient was consented by a nonstudy physician. Therefore, 42 prospectively enrolled patients were included in the analysis. Each patient's MWS was determined upon presentation, along with the following demographic data: age, gravity, parity, GA at presentation, whether there was a history of asthma, anxiety, cardiac disease, hypertension, or preeclampsia.

Since the number of patients with a documented PE was limited, data from a retrospective cohort were included in the study to capture all eligible patients who presented to the three hospitals from January 2009 to June 2014. A waiver of consent was used for accessing the charts of eligible patients with the ICD9-CM code 673.20 and 673.24 (obstetrical clot, embolism). Pregnant patients were included if a D-dimer was obtained at the time of diagnostic imaging (spiral CT or VQ scan). Based on chart review, 14 patients (23.7\%) had a documented PE in pregnancy and a D-dimer level available, so were included in the study. A MWS was assigned by one of the authors based on review of the electronic medical record. Therefore, the combined sample size of the prospective and retrospective cohorts was 59 .

Data are presented as mean \pm standard deviation or number (\%). The demographic and clinical outcomes were compared between the PE and no PE groups via chi-square test or Fishers exact test when categorical and via Student $t$-test when continuous. One way analysis of variance was performed to compare the D-dimer levels for the three trimesters, while Pearson correlation test was performed to confirm the relationships among D-dimer, MWS, and BNP scores. A two-tailed $p$ level of 0.05 was considered statistically significant in all analyses. The diagnostic properties including sensitivity, specificity, positive and negative predictive values of MWS, and D-dimer alone and combined were assessed. All analyses were performed using SPSS for Windows, version 20.0 (SPSS Inc., Chicago, IL).

\section{Results}

The final sample size of 59 women included 17 patients (28.8\%) who had evidence of $\mathrm{PE}$ ( 3 prospective and 14 retrospective) and 42 patients (71.2\%) who had no evidence of $\mathrm{PE}$ (all prospective). No significant differences were noted between the two patient groups regarding the studies demographic characteristics, 
e162 Diagnosing Pulmonary Embolism in Pregnancy Parilla et al.

Table 3 Demographic characteristics

\begin{tabular}{|c|c|c|c|}
\hline Variable & $\begin{array}{l}\mathrm{PE} \\
N=17\end{array}$ & $\begin{array}{l}\text { No PE } \\
N=42\end{array}$ & $p$-Value \\
\hline Age, mean (SD) & $30.1(6.3)$ & $29.6(4.4)$ & 0.705 \\
\hline Gestational age (wk), mean (SD) & $11.6(6.6)$ & $27.6(7.2)$ & $<0.001$ \\
\hline \multicolumn{4}{|l|}{ Chief complaint, $n$ (\%) } \\
\hline SOB & $9(52.9)$ & $17(40.5)$ & \multirow[t]{4}{*}{0.625} \\
\hline $\mathrm{CP}$ & $1(5.9)$ & $6(14.3)$ & \\
\hline SOB and $C P$ & $6(35.3)$ & $18(42.9)$ & \\
\hline Pain in other locations (except CP) & $1(5.9)^{\mathrm{a}}$ & $1(2.4)^{\mathrm{b}}$ & \\
\hline \multicolumn{4}{|l|}{ Parity, $n(\%)$} \\
\hline 0 & $1(6.3)$ & $19(45.2)$ & \multirow[t]{2}{*}{0.005} \\
\hline$\geq 1$ & $15(93.8)$ & $23(54.8)$ & \\
\hline Anxiety, $n(\%)$ & $17(100)$ & $38(90.5)$ & 0.314 \\
\hline Asthma, $n$ (\%) & $15(88.2)$ & $35(83.3)$ & 1.000 \\
\hline
\end{tabular}

Abbreviations: $\mathrm{CP}$, chest pain; $\mathrm{PE}$, pulmonary embolism; SD, standard deviation; SOB, shortness of breath.

${ }^{\mathrm{a}}$ Right shoulder and side.

bSubsternal and epigastric.

except the GA and parity ( - Table 3 ). The patients with evidence of PE had a significantly lower GA $(11.6 \pm 6.6$ vs. $27.6 \pm 7.2$ weeks, $p<0.001$ ). Parity categorized as 0 versus $\geq 1$ was statistically significant between the two groups, with more women with evidence of PE having a parity of $\geq 1$. None of the patients had cardiac disease, hypertension, or preeclampsia.

The clinical outcomes are reported in -Table 4. Patients in both groups had comparable heart rates and BNP levels. A BNP level was considered abnormal if $>100 \mathrm{pg} / \mathrm{mL}$. Since none of the study patients had abnormal BNP levels, echocardiography was not performed. Patients with evidence of PE had significantly higher D-dimer levels and MWS. When combining the D-dimer levels and MWS together, all women with evidence of PE had abnormal testing compared with only $11(26.2 \%)$ of the women with no evidence of PE $(p<0.001)$. The four abnormal MSW scores were secondary to "clinical signs of deep vein thrombosis (DVT)" in three patients, pulse $>100$ in two patients, and "other diagnosis less likely than PE" in one patient. Two patients had more than one abnormal criteria.

The diagnostic properties of D-dimer and MWS alone and combined are presented in -Table 5. The combination of a trimester-specific D-dimer level along with the MWS has a sensitivity of $100 \%$ and specificity of $73.8 \%$ in predicting PE if either was abnormal. The positive and negative predictive values were 60.7 and $100 \%$, respectively. No study patient was receiving anticoagulation at the time of presentation.

Table 4 Clinical outcomes

\begin{tabular}{|l|l|l|l|}
\hline Variable & $\begin{array}{l}\mathrm{PE} \\
\mathrm{N}=17\end{array}$ & $\begin{array}{l}\text { No PE } \\
N=42\end{array}$ \\
\hline Pulse $\geq 100, n(\%)$ & $4(23.5)$ & $18(42.9)$ & 0.164 \\
\hline MWS, mean (SD) & $2.9(1.3)$ & $1.1(1.1)$ & $<0.001$ \\
\hline MWS $\geq 4, n(\%)$ & $4(23.5)$ & 0 & 0.005 \\
\hline D-dimer, mean (SD) & $4.9(3.9)$ & $1.5(1.1)$ & $<0.001$ \\
\hline First trimester (13 vs. 2 patients) & $5.7(4.1)$ & $1(0.7)$ & \\
\hline Second trimester (3 vs. 11 patients) & $2.2(1.2)$ & $0.8(0.3)$ & $1.8(1.3)$ \\
\hline Third trimester (1 vs. 29 patients) & 2.8 & $21(12.1)$ & 0.163 \\
\hline BNP, mean (SD) & $34(25.5)^{\mathrm{a}}$ & 0.005 \\
\hline Abnormal MWS, $n$ (\%) & $4(23.5)$ & 0 & $<0.001$ \\
\hline Abnormal D-dimer, $n$ (\%) & $16(94.1)$ & $11(26.2)$ & $<0.001$ \\
\hline Abnormal MWS and/or D-dimer, $n(\%)$ & $17(100)$ & $11(26.2)$ & \\
\hline
\end{tabular}

Abbreviations: BNP, brain natriuretic protein; MWS, modified Wells score; PE, pulmonary embolism; SD, standard deviation. ${ }^{a} n=3$. 
Table 5 Diagnostic properties

\begin{tabular}{|l|l|l|l|}
\hline Metric & MWS & D-dimer & MWS + D-dimer \\
\hline Sensitivity (\%) & 23.5 & 94.1 & 100 \\
\hline Specificity (\%) & 100 & 73.8 & 73.8 \\
\hline $\begin{array}{l}\text { Negative predictive } \\
\text { value (\%) }\end{array}$ & 76.4 & 97.0 & 100 \\
\hline $\begin{array}{l}\text { Positive predictive } \\
\text { value (\%) }\end{array}$ & 100 & 59.3 & 60.7 \\
\hline
\end{tabular}

Abbreviation: MWS, modified Wells score.

Finally, significant correlations were noted between the MWS and D-dimer $(r=0.335, p=0.010)$ and between the Ddimer and BNP $(r=0.343, p=0.028)$. Although not statistically significant, a positive correlation was noted between the MWS and BNP $(r=0.299, p=0.059)$.

\section{Comment}

The identification of PE in pregnancy presents a diagnostic dilemma. Although physiologic changes of pregnancy often mimic those of PE, it is not obvious which patient has a benign finding and which has a life-threatening diagnosis. The mortality rate of undiagnosed PE in pregnancy approaches 15 to $30 \%,{ }^{1}$ thus a prompt diagnosis is essential. The gold standard for diagnosis includes either a CTPA or VQ scan, which results in radiation exposure to both the mother and fetus. Both D-dimer levels and the MWS have been proven in large prospective studies to be reliable screening methods for PE outside of pregnancy. However, there have been no prospective studies to evaluate the use of these screening modalities in pregnant women, owing to the relatively small number of women diagnosed with PE. Due to the obvious lack in dependable screening tools for this unique population, there is a heavy reliance on diagnostic imaging modalities. ${ }^{11}$

D-dimers are plasma breakdown products of cross-linked fibrin and therefore are used as markers of recent thrombus formation. They are also elevated in malignancy or acute inflammatory states. Therefore, they are used for their negative predictive value. Chan et $\mathrm{al}^{6}$ hypothesized that higher cutoff points for the diagnosis of DVT may compensate for the higher baseline $\mathrm{D}$-dimer values that are seen in pregnancy. To our knowledge to date, this is the first study to look at trimester-specific D-dimer levels and the MWS to triage pregnant women into a low-risk category for PE. We identified that using both trimester-specific D-dimer levels and the MWS score was $100 \%$ sensitive and $81.4 \%$ specific in detecting PE if either was abnormal. Thus, all women with PE $(n=17)$ would have received imaging with the use of this screening modality and we could have safely decreased the number of women imaged by $52.5 \%$.

It is also important to recognize that the positive predictive value of this screening modality is $60.7 \%$. This means that $39.3 \%$ of the time when a patient screens positive, the patient will not actually have a PE. Therefore, it is imperative to proceed with diagnostic imaging when a screen is positive rather than reflexively treating.

We were interested in seeing if BNP levels were also useful in evaluating patients with signs or symptoms suggestive of PE as BNP was found to be useful in the detection of acute PE in postsurgical patients, where D-dimer levels are frequently elevated and therefore not discriminatory. ${ }^{9,10}$ BNP was not helpful in discriminating between low and high risk for PE in this study as all of the BNP levels were within the normal range ( $<100 \mathrm{pg} / \mathrm{mL}$ ). The number of BNP levels collected was also small $(n=24)$ as they were only obtained from the prospectively enrolled patients, only three of whom had a documented PE. We did, however, identify a statistically significant correlation between the D-dimer and BNP. Thus, trimester-specific BNP levels may potentially prove useful in the future.

Limitations of this study include a relatively small number of enrolled patients, although this did represent the "real-life" experience in three large tertiary hospitals with more than 10,000 deliveries per year. In addition, the need to add a retrospective arm meant that assignment of the MWS was based on chart review, thus information necessary to accurately assign a score may not have been available. Based on our experience of assigning a MWS, we noted that using the subjective criteria of "other diagnosis less likely than PE" is confusing for the practitioner and may result in a less accurate MWS. We advocate using alternate wording such as "PE is the most likely diagnosis."

In conclusion, our findings suggest that the MWS in combination with trimester-specific D-dimer levels may be useful to triage pregnant women into a low-risk category for PE and thus decrease radiation exposure to mothers and their fetuses. However, before adopting this strategy, larger, confirmatory trials are needed.

\section{Acknowledgments}

This study was financially supported by Small Research Grant Program, James R. \& Helen D. Russell Institute for Research \& Innovation, Advocate Lutheran General Hospital, Park Ridge, IL

\section{Note}

Registered with clinicaltrials.gov \# NCT02709174.

\section{References}

1 O'Connor C, Moriarty J, Walsh J, Murray J, Coulter-Smith S, Boyd W. The application of a clinical risk stratification score may reduce unnecessary investigations for pulmonary embolism in pregnancy. J Matern Fetal Neonatal Med 2011;24(12):1461-1464

2 Wells PS, Hirsh J, Anderson DR, et al. Accuracy of clinical assessment of deep-vein thrombosis. Lancet 1995;345(8961): 1326-1330

3 Wells PS, Anderson DR, Rodger M, et al. Excluding pulmonary embolism at the bedside without diagnostic imaging: management of patients with suspected pulmonary embolism presenting 
e164 Diagnosing Pulmonary Embolism in Pregnancy Parilla et al.

to the emergency department by using a simple clinical model and d-dimer. Ann Intern Med 2001;135(2):98-107

4 Cutts BA, Tran HA, Merriman E, et al. The utility of the Wells clinical prediction model and ventilation-perfusion scanning for pulmonary embolism diagnosis in pregnancy. Blood Coagul Fibrinolysis 2014;25(4):375-378

5 Kline JA, Williams GW, Hernandez-Nino J. D-dimer concentrations in normal pregnancy: new diagnostic thresholds are needed. Clin Chem 2005;51(5):825-829

6 Chan WS, Lee A, Spencer FA, et al. D-dimer testing in pregnant patients: towards determining the next 'level' in the diagnosis of deep vein thrombosis. J Thromb Haemost 2010;8(5):1004-1011

7 Abbassi-Ghanavati M, Greer LG, Cunningham FG. Pregnancy and laboratory studies: a reference table for clinicians. Obstet Gynecol 2009;114(6):1326-1331
8 Tanous D, Siu SC, Mason J, et al. B-type natriuretic peptide in pregnant women with heart disease. J Am Coll Cardiol 2010; 56(15):1247-1253

9 Winkler BE, Schuetz W, Froeba G, Muth CM. N-terminal prohormone of brain natriuretic peptide: a useful tool for the detection of acute pulmonary artery embolism in post-surgical patients. Br J Anaesth 2012;109(6):907-910

10 Coutance G, Le Page O, Lo T, Hamon M. Prognostic value of brain natriuretic peptide in acute pulmonary embolism. Crit Care 2008; 12(4):R109

11 Kline JA, Richardson DM, Than MP, Penaloza A, Roy PM. Systematic review and meta-analysis of pregnant patients investigated for suspected pulmonary embolism in the emergency department. Acad Emerg Med 2014;21(9): 949-959 